\title{
Simulation Studies on the Lipid Interaction and Conformation of Novel Drug Delivery Pseudopeptidic Polymers
}

\author{
Shuzhe Wang and Fernando Bresme* \\ Department of Chemistry, Imperial College London, SW7 2AZ, London, United Kingdom \\ E-mail: f.bresme@imperial.ac.uk
}

\begin{abstract}
A class of pseudopeptides based on poly(lysine iso-phthalamide) backbone have emerged as promising drug delivery candidates due to their $\mathrm{pH}$ activated membrane disruption ability. In order to gain molecular understanding on these novel polymeric species, we have constructed force field parameters and simulated the behaviours of polymers with and without phenylalanine grafted as side chains at conditions compatible with different $\mathrm{pH}$. The free energy changes upon polymer permeation through membrane were calculated using the umbrella sampling technique. We show that both polymers with and without grafts interact better with the membrane at conditions compatible with lower $\mathrm{pH}$. The conformational states of the polymers were investigated in water and at a water-membrane interface. Based on Markov state modelling results, we propose a possible advantage of grafted polymer over un-grafted polymer for membrane rupture, due to their quicker conformational rearrangement kinetics.
\end{abstract}




\section{Introduction}

The design of effective therapeutic agents is a primal interest of the pharmaceutical sector. It channels high level of investments into researches at many fronts, including the identification of new effectable therapeutic targets. ${ }^{1}$ However, when a target is intracellular, getting the drug through the cell membrane is challenging - drug delivery molecules can assist with this. ${ }^{[2]}$ Depending on how the delivery vehicles transport the drug cargo, there are two main delivery strategies. One type assembles into micelles which encapsulates drug candidate like viral vec-

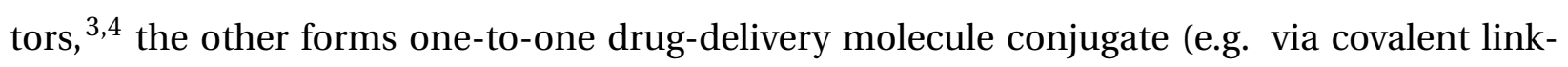
age) ${ }^{5[6]}$ In this work we study a class of pseudopeptides belonging to the latter type.

The pseudopeptides are inspired by membranelytic fusogenic viral peptide haemagglutinin. ${ }^{7}$ The first synthesised species had lysine isophthalamide as monomer unit to form a linear polymer (Fig 1). Further works performed by Chen et al. found that grafting different hydrophobic amino acids such as valine, leucine and phenylalanine enhanced membranelytic ability.

The polymers formed have number-average molecular mass $M_{n}=17900$ - corresponding to $\sim 65$ repeat units. ${ }^{10} \mathrm{~A}$ vital feature of these polymers is their $\mathrm{pH}$ activated membrane rupturing ability due to protonation of carboxylate sites. The proposed mechanism of action has the poly-

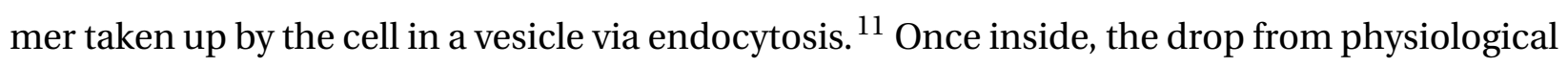
pH ( 7.4) to endosomal pH ( 5.5) protonates the initially negatively charged polymer. The polymer then ruptures the vesicle before being sent for lysosomal disposal, therefore delivering its cargo freely to cytoplasmic targets.

Numerous experimental techniques have been applied to study the $\mathrm{pH}$ and grafting effects on the pseudopeptides. ${ }^{8-10 \mid 12}$ Being pseudopeptidic, the polymers have minimal safety concerns. Biocompatibility tests using alamar blue assay showed the polymers are not cytotoxic up to $1 \mathrm{~g} \mathrm{~mL}^{-1}$. Using fluorescence spectroscopy, it was found the fluorescence signal of pyrene fluorophore rapidly dropped upon reducing the $\mathrm{pH}$. This was claimed to be due to change of polymer conformation from extended chains to collapsed structures that enveloped the fluorophore. The authors also found this effect was less pronounced with lower polymer concentra- 


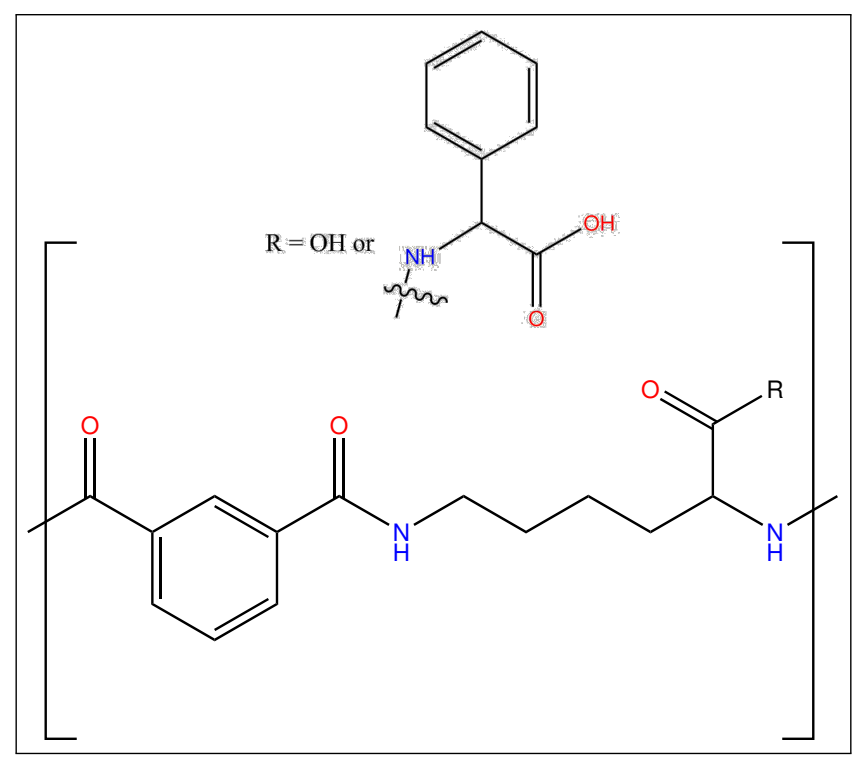

Figure 1: Poly(lysine isophthalamide) monomer unit and phenylalanine grafted monomer unit. $\mathrm{R}=\mathrm{OH}$ represents the unmodified poly(lysine isophthalamide) and the other fragment corresponds to the phenylalanine graft.

tion which suggest this to be a cooperative effect. Optical density was measured to determine the $\mathrm{pH}$ values where polymers precipitate from aqueous solution. It was found the precipitation $\mathrm{pH}$ can be modulated by amino acid grafting. The $\mathrm{pH}$ for precipitatiom increases with the grafting of the hydrophobic side chains. In cell assays, both hemolysis assay (using red blood cell) and laser scanning confocal microscopy (using HeLa cell) showed that at physiological $\mathrm{pH}$ the polymers are non-lytic. While below pH 5.5, unmodified poly(lysine isophthalamide) had limited hemolytical activity while grafted polymers displayed significant higher lysing with increasing degree of grafting. It was hypothesised the observation was due to the grafted polymer having more hydrophobic sites for polymer adsorption to membrane head groups and forming more hydrophobic structures which favours entering via the tail region.

However, due to the limited resolution of the aforementioned experimental techniques, a molecular understanding of the pseudopeptides interaction with water as well as with lipid membrane is lacking. For this, computational modelling and Molecular Dynamics (MD) simulation of the modelled systems can offer validation and insight. It is our intention to initiate such in silico investigation here. 
Although there is no computational study on these pseudopeptides, other polymeric species for drug delivery purpose have been computationally analysed and comprehensive reviews have been written. ${ }^{13 \mid 14}$ In recent years, Choudhury et al. studied how poly(ethylenimine), which is capable of forming polycations at lower $\mathrm{pH}$, interacts with membrane bilayers. ${ }^{15}$ They ran separate simulations for the protonated and deprotonated polymers, positioned at the water phase, lipid core and water-lipid interface to investigate different stages of the membrane disruption process. From the simulation trajectories, they observed the polymer being more elongated in the protonated form. In addition, they observed disruption of the membrane integrity by the polycationic form only. A similar class of polymers was studied by Kepczynski et al. ${ }^{16}$ Instead of running simulations with polymers positioned at different places, they selected a much shorter oligomer to mimic the polymer and started simulations by placing it in water outside of lipid bilayer. They directly observed cationic oligomer penetration process which aligned with experimental measurements. Kim et al. 17 attempted to understand how a much bulkier polyamidoamine dentrimeric polymer interacts with the membrane. This followed from previous coarse grained ${ }^{18}$ MD by Lee et al. ${ }^{19}$ Due to the size of the polymer, they only observed polymer adsorption onto the bilayer. Using the trajectories, they elucidated the conformational change of the polymer upon adsorption using parameters such as the radius of gyration. Membrane penetration mechanisms of poly(ethylene oxide), poly(propylene oxide) and their copolymers were studied by Nawas et al. ${ }^{20}$ Focusing on structure descriptors of the model membrane such as area per lipid and head group orientations, they inferred the hydrophilic regions in the copolymers interact well with lipid head groups, resulting in pore-forming modification of the membrane structure. Meanwhile, Samanta et al. calculated free energy of lipid permeation by these polymers to assess preferential interaction based on varied polymer compositions. ${ }^{21}$ They discovered the energy barrier for poly(propylene oxide) lipid penetration was smaller than that of poly(ethylene oxide), and that poly(propylene oxide) feature longer residence times. Unlike previous studies that only used conventional MD, they demonstrated that steered MD method was needed to sufficiently sample the process for even small dimeric and 
trimeric polymer penetration.

Here, we model the un-grafted poly(lysine isophthalamide) in different $\mathrm{pH}$ environments using different level of protonation. The same is done for the grafted polymer. For the grafting, we selected to model phenylalanine because it was the most hydrophobic side chain tested experimentally ${ }^{9}$ and thus should offer the greatest contrast to the un-grafted form. Using steered $\mathrm{MD}$, the free energy change during the membrane penetration process will be mapped to understand the experimentally observed membrane-lysis results. Further, Markov State Models (MSM) are employed to identify key conformational states of the polymer fragments, in different environments, namely, membrane surface and bulk solution.

The paper is structured as follows: the main computational techniques used are described first. Then, findings are reported and discussed before concluding remarks.

\section{Methods}

\subsection{Simulation Parameters}

The simulation models were parametrised by the united atom force field GROMOS 53a6. ${ }^{22]} \mathrm{We}$ used a dipalmitoylphosphatidylcholine (DPPC) lipid bilayer as model for the plasma membrane. Although this is a gross approximation, it is one adopted by many simulational studies due to the abundance of DPPC in biological membranes. ${ }^{19220123}$ Topology and configuration of DPPC bilayer (64 lipids per layer) were downloaded from reference. ${ }^{[24}$ The SPC/E water model was used ${ }^{25}$ In order to better approximate the experimental conditions, $0.1 \mathrm{M}$ solution of $\mathrm{NaCl}$ salt (using GROMOS 53a6 native parameters) was added to mimic the physiological conditions and the buffers used in the experiments. 26

The time step for the simulations was chosen to be 2 fs. Unless specified otherwise, each system was subjected to 500 ps of NVT equilibration and 500 ps NPT equilibration before the production runs in the NPT ensemble. The simulations were performed at $310 \mathrm{~K}$ using the v-rescale thermostat ${ }^{27}$ with a coupling constant of $0.1 \mathrm{ps,} \mathrm{and} \mathrm{at} \mathrm{constant} \mathrm{pressure} \mathrm{using} \mathrm{the}$ 
Parrinello-Rahman barostat $\frac{28 \mid 29}{2}$ with a coupling constant of 2 ps and compressibility of $4.5 \times$ $10^{-5}$ bar $^{-1}$. The LINCS algorithm ${ }^{30}$ was used to constrain all bonds. Short-range non-bonded were computed using a cut-off of $1.0 \mathrm{~nm}$ and the long-range electrostatic interactions were computed using the Particle Mesh Ewald (PME) method ${ }^{31}$ with $0.16 \mathrm{~nm}$ for the Fourier spacing. For simulations in bulk (without the lipid bilayer), there is symmetry in all three dimensions and the pressure coupling type was set to isotropic. For simulations of lipid bilayers, we set the barostat to semi-isotropic with the $\mathrm{z}$-direction decoupled from $\mathrm{x}$ and $\mathrm{y}$. In this case the pressure normal to the bilayer plane was set to $1 \mathrm{bar}$ and the surface tension to $0 \mathrm{mN} / \mathrm{m}$, to achieve a tensionless state. Simulations involving charged polymers require the addition of counterions to achieve an electroneutral system. In this case and due to the use of periodic boundary conditions there is a possibility of building up an excess of charge on one side of the membrane, leading to transient electrostatic fields and that can induce membrane poration. To prevent this effect we employed the approach discussed in reference. ${ }^{32}$ In this method two walls with 9-3 type Lennard-Jones interactions are set at the edges of the box, to prevent the charge accumulation. The interactions of the 9-3 potential were obtained using the LennardJones interaction strength of the oxygen in the water molecules (SPC/E), with the wall density set to $110 \mathrm{~nm}^{-3}$ and the interpolation order of the PME method was set to 3 .

Molecular dynamics simulations were performed using GROMACS v.5.1.4. $\frac{33-35}{-35}$ and the systems were visualised using VMD ${ }^{36}$ All simulations were submitted to the high performance cluster at Imperial College London. $\frac{37}{37}$

\subsection{Force Field Construction}

As there is no reported computational study of either un-grafted or grafted poly(lysine isophthalamide), their topologies were constructed first. An intuitive way is to devise the parameters for a single repeat unit of the two kinds of monomers, which can then be concatenated to make polymers of arbitrary compositions.

The polymers are denoted pseudopeptides because they have amino acid components. There- 
fore it is feasible to construct the monomers using parameters from the GROMOS 53a6 amino acid database, which have parameters for lysine and phenylalanine and can aid parametrising the isophthalamide part.

Figure 2 shows the final atom names assigned to each atom in the two monomer types. Hereafter, we denote the un-grafted monomer unit type A, while the grafted monomer type $\mathbf{B}$, respectively assigned either blue or magenta in figure 2 .

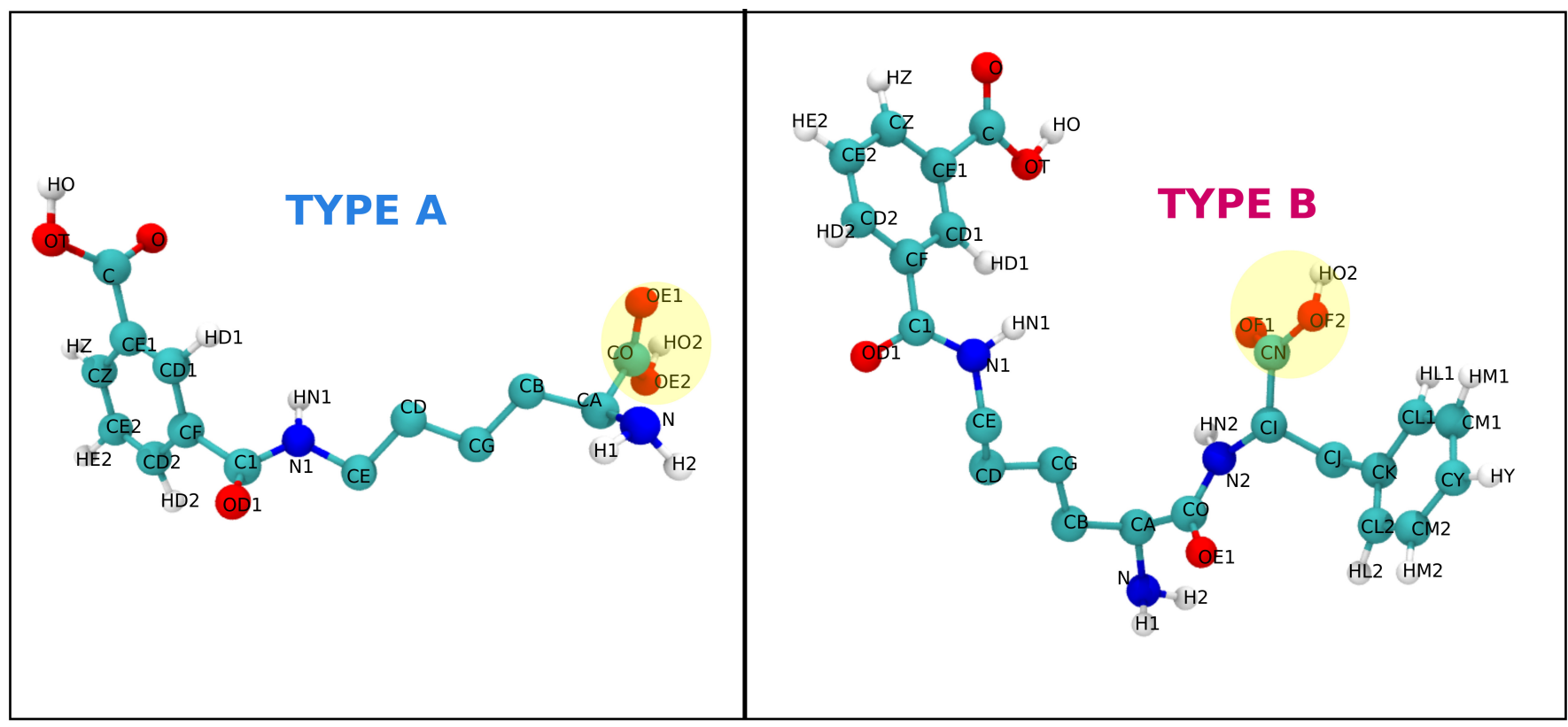

Figure 2: Unique atom names assigned to the monomers used in the force field. The atom names for the backbone (N-CA-CB-....-CE1-C-O) is the same for both monomer. Yellow shaded region indicates the sites of (de-)protonation. The colour blue is associated to type A monomer and magenta to $\mathrm{B}$ in this study.

For new bonds/angles/torsions needing definitions in the monomers, the most suitable ones within the amino acid database were picked. ${ }^{22}$ See supplementary information for further detail.

It was decided to define two monomers, each with two protonation states: the neutral form and -1 charged form, akin to how amino acids with multiple charge states are treated in the GROMOS 53a6 amino acid database. ${ }^{[22}$ The circled region in Fig. 2 are the (de-)protonation sites. GROMACS treats protonation state selection only for a hard-coded list of natural amino 
acids. Hence for our computations we defined four new monomers: the protonated, charge neutral forms of the two monomers were called $\mathrm{A}(0)$ and $\mathrm{B}(0)$. The deprotonated, negatively charged monomers were assigned A(-) and B(-). See supplementary information for the force fields of these four monomers.

To obtain polymers with different monomer combinations, their force fields can be robustly created by GROMACS's pdb2gmx tool. ${ }^{33}$ Because the monomer parameters were incorporated into the amino acid database, pdb2gmx can automatically generate the topology when provided an initial configuration. To construct the configuration file, a Python script that manipulated the coordinates of individual monomers was written. It can put together any combinations of the monomer units to give an extended conformer (Fig. 3). The figure below shows a $\mathrm{B}(0)$ monomer unit joined on either side by 2 units of $A(0)$ monomer. Such a polymer can then be solvated and minimised to give more energetically favoured conformation before further simulation. Five repeat unit polymers were chosen for the following studies. The extended form of the BBBBB polymer is shorter than the side length of the lipid bilayer ( $\sim 6.3 \mathrm{~nm}$ long), to eliminate issues concerned with the self-interactions due to periodic boundary condition.

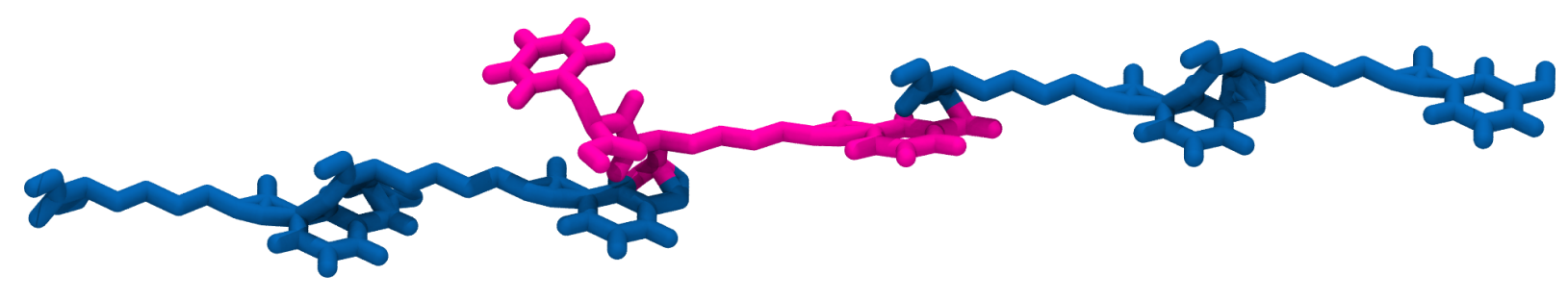

Figure 3: A stretched polymer chain with 5 repeat units : $\mathrm{A}(0)$ in blue, $\mathrm{B}(0)$ in magenta. A script (see supplementary information) allows the easy construction of polymers of varied compositions. This polymer contains two type $A(0)$ monomers in blue sandwiching a $B(0)$ monomer in magenta. 


\subsection{Free Energy Computations}

Umbrella Sampling (US) was employed to calculate the potential of mean force by pulling the polymer from bulk water to the center of the lipid along the direction of the lipid bilayer normal plane with a pulling rate of $0.005 \mathrm{~nm} \mathrm{ps}^{-1}$. From the pulling trajectory, frames were selected with $0.2 \mathrm{~nm}$ apart along the reaction coordinate as starting simulation windows. Windows were simulated in parallel where the polymers were constrained at the center of each window using a harmonic constrain force with force constant of $3000 \mathrm{~kJ} \mathrm{~mol}^{-1} \mathrm{~nm}^{-2}$. Each window was equilibrated for $1 \mathrm{~ns}$ before simulating for $30 \mathrm{~ns}$ for configurational sampling. The potential of mean forces (PMF) were constructed using weighted histogram analysis method (WHAM) and errors were estimated using Bayesian bootstrapping, via GROMACS 5.1.4 implementation. 33

\subsection{Markov State Model (MSM)}

We applied MSM to identify low energy conformations and calculate long term kinetics of the

polymers. MSM is a recent advancement in quantitative analysis of MD data. ${ }^{38 \sqrt{39}}$ The MD trajectories are approximated as memory-less system where the probability distribution of future states is only governed by the current state (no past states required) as it follows from a Markov chain process.

To generate a MSM, the following sequence of steps needs to be applied to a system. First, we selected the atom coordinates $\mathbf{r}(t)$ as descriptor of the time evolution of the polymer structures.

\section{Time-lagged independent component analysis (TICA)}

The number of dimensions for the selected descriptors are usually unnecessarily large, which could lead to over-fitting. TICA is a common dimensional reduction technique for analysing time evolution of data. $\underline{40 \mid 41}$

A covariance matrix $\mathbf{C}(\tau)$ is built based on all pairs of descriptor vector $\mathbf{r}(t)$ separated by $\tau$ time steps. When there are $\mathrm{M}$ descriptors included in the analysis and for a trajectory with $\mathrm{N}$ 
frames, the covariance matrix $\mathbf{C}(\tau)$ has dimensions $M \times(N-\tau), c_{i j}$ is a particular element within the matrix:

$$
c_{i j}(\tau)=\frac{1}{N-\tau-1} \sum_{t=1}^{N-\tau} r_{i}(t) r_{j}(t+\tau)
$$

Eigenvalue decomposition is applied to the resultant matrix:

$$
\mathbf{C}(\tau)=\mathbf{U} \Lambda \mathbf{U}^{-1},
$$

where $\mathbf{U}$ is the eigenvector matrix and $\Lambda$ the eigenvalue diagonal matrix (eigenvalues ordered from the largest to smallest). To reduce the dimension from $M$ to a smaller $m$, the time dependent positions, $\mathbf{r}(t)$, were multiplied by the first $m$ columns of the eigenvector matrix $\left(\mathbf{U}_{\mathbf{m}}\right)$ to get a new vector $\mathbf{z}(t)$ that has less dimensions.

$$
\mathbf{z}(t)=\mathbf{r}(t) \mathbf{U}_{\mathbf{m}}
$$

In comparison to other methods such as principle component analysis, TICA can identify better slow reaction coordinates, which are key for identifying stable polymer conformations.

\section{Regular space clustering}

To sufficiently define transitions between different coordinates in $\mathbf{z}(t)$, the finite amount of simulation data needs to be aggregated into discrete conformation states. This discretisation of trajectories is often done using $\mathrm{k}$-means clustering ${ }^{42}$. It starts by randomly selecting $\mathrm{k}$ different coordinates as the cluster centers. Then, it repeatedly (for a given number of iterations) assigns the remainder coordinates to the center that is closest to them in terms of distances and updates the centers as mean positions of all the coordinates belonging to the same cluster.

\section{Transition matrix and implied time-scale}

After space discretisation, a transition matrix was determined. Here, a new lag time $\tau_{0}$ that is different to the lag time $\tau$ used during TICA was needed. A transition matrix $\mathbf{P}\left(\tau_{0}\right)$ based on 
this lag time can be created, where an element $p_{i j}$ can be estimated by the normalised count of transitions from the cluster $i$ at a given time to another cluster $j$ after $\tau_{0}$ times. ${ }^{43}$

An implied time-scale is the decay time of a physical process. The most dominant eigenvalues in the transition matrix correspond to the slowest decay processes in the system, which in turn connects stable conformations. Formally, for the $i$ th slowest decay process:

$$
t_{i}\left(k \tau_{0}\right)=\frac{k \tau_{0}}{\ln \lambda_{i}\left(k \tau_{0}\right)}
$$

where $k$ is an arbitrary positive integer and $\lambda_{i}$ the $i$ th eigenvalue and $t_{i}$ the $i$ th implied time of decay. Because the decay time is an inherent property of the system, it should not be a function of the lag time, as it is arbitrarily user-defined. When the system is Markovian, $\lambda_{i}\left(k \tau_{0}\right)=\lambda_{i}\left(\tau_{0}\right)^{k}$ and this would make the implied time independent to the lag time as $k$ in the denominator and nominator cancels. This manifests in the time of decay remaining invariant upon constructing transition matrix with longer lag times, see figure ?? in supplementary information for an example plot analysis. Therefore, implied time-scale served as a validation that a suitable lag time has been picked for the transition matrix so the system behaves Markovian.

\section{Perron-cluster cluster analysis (PCCA)}

PCCA outputs long-lasting conformational states. It projects the discrete coordinates along the first $n$ user-defined eigenvector directions. ${ }^{44}$ When one eigenvector is used, the projected coordinates all lie on a line that is bifurcated to give two metastable coordinate sets. And when $n$ eigenvectors are chosen for projection, $n+1$ metastable structure sets are produced. In this way, PCCA aggregates structures in the discrete state space into a few metastable sets, each such set $S_{i}$ represents a group of structures where the polymer fragment stay for significant time before jumping to another set $S_{j}$. Thus, each set can be associated with a free energy basin.

The sophisticated techniques detailed above are contained in a robust and well-documented Python framework called pyEMMA, which was utilised for the analysis reported in this work. ${ }^{45}$ 


\section{Results and Discussion}

\subsection{Potential of Mean Force (PMF)}

\section{Monomers}

The free energy changes of the four different monomers $\mathrm{A}(0), \mathrm{A}(-), \mathrm{B}(0)$ and $\mathrm{B}(-)$ penetrating DPPC were calculated first (each window simulated for $20 \mathrm{~ns}$ ). Fig. 4 shows the PMF paths of the four species travelling from water to the middle of the membrane (left to right), with the vertical grey line indicating the average position of lipid head groups (choline region in the bilayer). The free energies of monomers in water have been assigned to zero for ease of comparison.

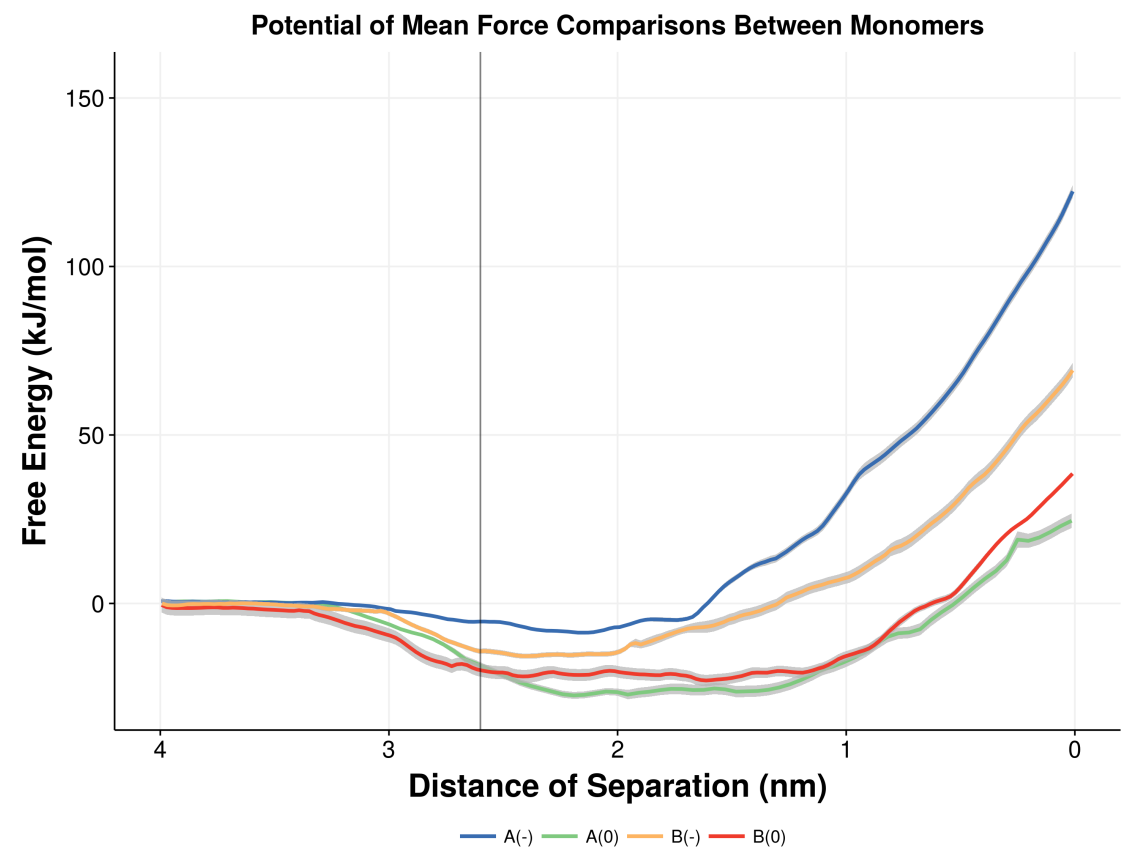

Figure 4: PMFs for various monomer types penetrating the DPPC bilayer. Semi-transparent grey colour surrounding each PMF line indicates the statistical error bounds in WHAM estimation. The vertical line at $2.6 \mathrm{~nm}$ represents the average position of the center of mass of the choline head groups. Regions to the left of the vertical line corresponds to the water phase and regions to the right of the vertical line corresponds to the lipid phase. A distance of $0 \mathrm{~nm}$ on the $\mathrm{x}$-axis indicates the center of the lipid bilayer.

For all monomers, the interactions between monomers and lipid head group are energetically favourable irrespective of the charge state. While the free energies monotonically raise 
after entering the tail region and are less energetically favourable compared to that in water phase. The energy difference between the monomers at their minima is much less than the difference at the bilayer center. In all cases, the energies required to reach the bilayer center are at least $10 k T$. Therefore it is unlikely to observe such transition using unbiased MD.

Table 1 lists the energy maxima, minima and their difference along the path shown in the figure. Monomers with lower energy minima also have lower energy maxima. The trend observed can be attributed to the combination of two factors: electrostatic and hydrophobic interactions. It is less favourable for charged monomers to enter DPPC compared to the uncharged monomers. This suggests although the positively charged choline group in DPPC presents favourable interaction with $\mathrm{A}(-)$ and $\mathrm{B}(-)$, this is overshadowed by the electrostatic interaction with the negatively charged phosphate oxygen. The lower minima of $\mathrm{B}(-)$ than $\mathrm{A}(-)$ is likely because its pendant side chain can better shield the charge. For $A(0)$ and $B(0)$, charge neutrality diminishes the electrostatic effects and contributes to better interaction with the lipid head group. The highly lipophilic DPPC tail region is electrostatically unfavourable for the charge monomers and results in a much larger energy elevation than the neutral monomers. This is especially the case with the less protected charge of A(-), hence its highest observed energy. In their neutral forms, hydrophobic crowding favours the smaller $A(0)$ and thus its lower energy than $\mathrm{B}(0)$. For the monomers, the energy is mainly controlled by the level of electrostatic interaction.

\begin{tabular}{|c|c|c|c|c|}
\hline Free Energy $(\mathrm{kJ} / \mathrm{mol})$ & $\mathrm{A}(0)$ & $\mathrm{A}(-)$ & $\mathrm{B}(0)$ & $\mathrm{B}(-)$ \\
\hline Min & $-27.3 \pm 1.0$ & $-8.66 \pm 0.4$ & $-22.9 \pm 2.0$ & $-15.6 \pm 0.8$ \\
\hline $\operatorname{Max}$ & $24.5 \pm 2.1$ & $122 \pm 1.9$ & $38.5 \pm 0.2$ & $69.1 \pm 2.1$ \\
\hline Difference & $51.8 \pm 2.4$ & $131 \pm 1.9$ & $61.4 \pm 2.0$ & $84.8 \pm 2.3$ \\
\hline
\end{tabular}

Table 1: Free energy summaries for monomers in both protonated and deprotonated states, showing the free energy minima reached at the water-membrane interface, the maxima towards the bilayer center and the difference between the maxima and minima. 


\section{Polymers}

PMFs were calculated for polymers consisting of five repeat units of monomers. Such strands can be modelled as segments of experimentally tested pseudopeptides. We studied the free energy paths of poly(lysine isophthalamide) with different levels of phenylalanine grafting, which is modelled by replacing some type A with type B. We prepared polymer with pure type A (no grafts), namely AAAAA; and one B replacement of A at two different sites - AABAA and AAAAB; and polymer BBBBB. AABAA and AAAAA were designed to mimic polymer with low level of grafting, as $25 \%$ replacement was when marginal improvement in membranelysis started to be seen and the BBBBB chain was to mimic higher percentage of grafting. ${ }^{8}$

To approximate intracellular and physiological pH conditions, we simulated fully deprotonated and protonated polymer strands. Because the state of all monomers are the same (no partial protonation), we do not need to attach a charge state label to each monomer. Instead we can assign an overall state "0" if it is fully neutral and a state "-" if it is fully deprotonated (negatively charged). We used simulation with walls (see Methods section) for the charged polymers in order to prevent build up of counterions on one side the membrane and the resulting electrostatic fields, which can induce the nucleation of pores in the membrane. $\frac{32}{}$

We show in Fig. 5 that some trends are the same as in the monomer case. The strands all experience favourable interaction with the lipid head group and the free energy increases upon entering the lipid tail. The energy maxima reached by the charged polymers at the bilayer center are all higher than those reached by the neutral ones. While the neutral polymers all have lower free energy minima (below dashed line on the right half zoom-in) compared to the deprotonated ones.

The PMFs show the onset of favourable interaction between the lipid head group and BBBBB() is the earliest along the pathway. This is most likely due its bulkier polymer size. Generally, AABAA and AAAAB paths correspond more closely to that of the full A type polymer than full B type. This suggest increasing level of grafting manifests in gradual transition on the free energy trajectory. Intuitively, grafting the middle monomer (resulting in AABAA) will have a larger in- 
fluence on how the polymer strand packs as compared with grafting a terminal monomer. We indeed see that the results for AAAAB follow the polymer AAAAA closer than AABAA. However, it is interesting to see how AABAA(-) achieves a lower maxima towards the membrane core region.

\section{Potential of Mean Force Comparisons Between Polymers}
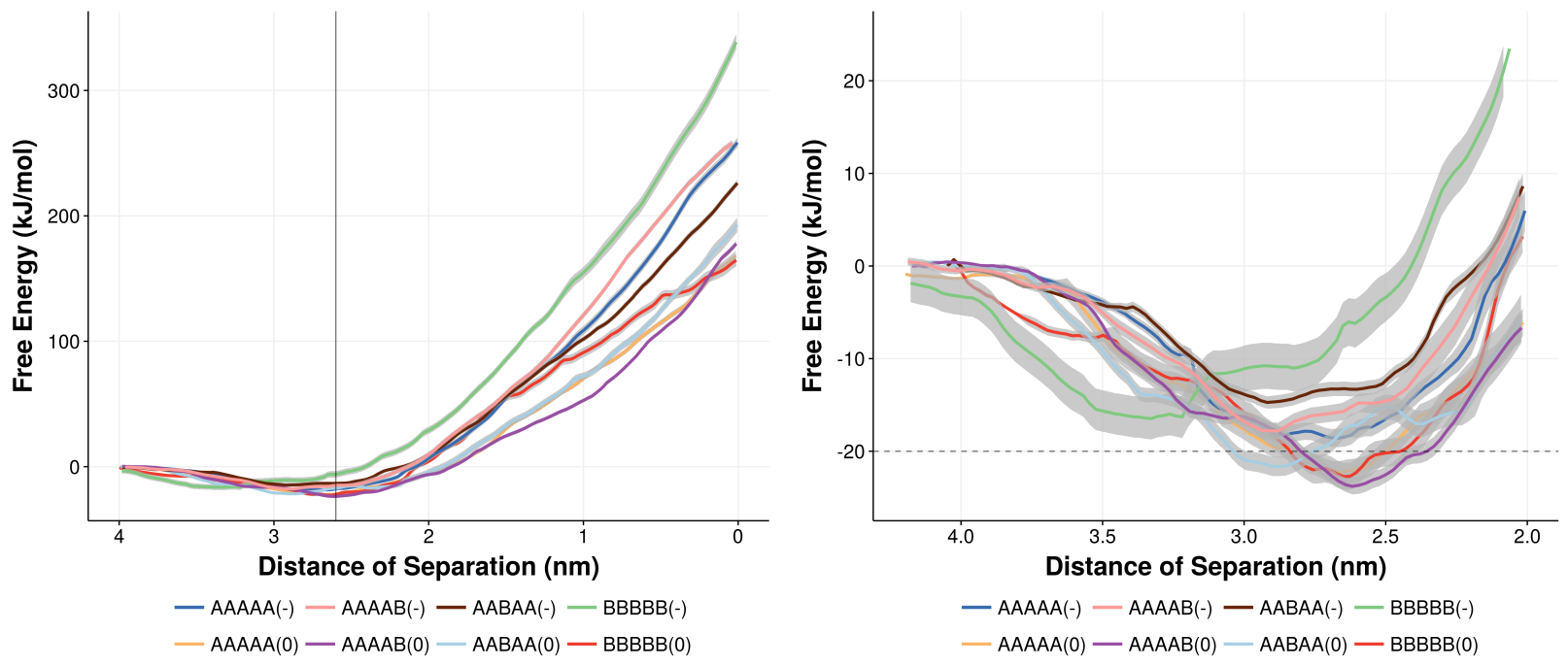

Figure 5: PMFs for 5 repeat unit polymers of various compositions penetrating the DPPC bilayer. Semi-transparent grey colour surrounding each PMF line indicates the statistical error bounds in WHAM estimation. The vertical line at $2.6 \mathrm{~nm}$ on the left plot represents the average position of the center of mass of the choline head groups. Regions to the left of the vertical line correspond to the water phase and regions to the right of the vertical line correspond to the lipid phase. A distance of $0 \mathrm{~nm}$ on the $\mathrm{x}$-axis indicates the center of the lipid bilayer. The horizontal dashed line on the right plot indicates free energy minima for charged ("-" state) and uncharged ("0" state) polymer species.

Table 2 lists the energy maxima, minima and their differences along the free energy path. Compared to the monomers, the level of favourable interaction with the head group has not markedly changed with increased polymer size, while the maxima at the center of the bilayer has risen significantly. The protonated polymers experience lower energy minima than charged ones and the overall free energy differences do not vary greatly with their composition. This is not the case for the charged polymers. Due to the heightened maxima, compositional change results in much greater change in free energy. Contrary to the monomers, $\mathrm{BBBBB}(-)$ has a higher maxima than AAAAA(-). This could mainly be attributed to two changes. Unlike in the monomer case where the charge of A is poorly protected, having five repeat units estab- 
lishes a backbone that is capable of shielding the negative charges from the lipid tails even without the help of pendant side chains of $\operatorname{BBBBB}(-)$. In addition, the negative charges create intramolecular electrostatic repulsion between the monomer units which swells the polymers when compared to their neutral forms. The pendant groups of BBBBB(-) will make the polymer bigger than AAAAA(-) and thus cause larger unfavourable hydrophobic crowding. Advancing the discussion below these changes are confirmed by the radius of gyration, which is bigger for $\operatorname{BBBBB}(-)$.

The results here support the $\mathrm{pH}$ modulated membranelytic capability observed in experiments. ${ }^{8 \mid 9}$ At physiological pH, polymers are in the charged state ("-" state above) and due to the higher free energy penalties do not exhibit membrane penetration. The drop in $\mathrm{pH}$ upon entering endosome results in de-ionisation. As seen, when fully protonated, the free energy barrier drops significantly which suggest easier passage. The PMFs indicate the $\mathrm{pH}$ effect predominately changes the interaction with the tail region where the energy drop upon protonation can be over $100 \mathrm{kJmol}^{-1}$. The fully grafted polymer BBBBB has the most pronounced $\mathrm{pH}$ mediated change as seen from the largest energy difference between fully protonated and charged states, making it the most controllable polymer. However, the PMFs suggest that at the fully neutral state, increased level of hydrophobic side chains does not energetically facilitate grafting onto the membrane head group or contribute to formation of hydrophobic domains that better interact with the bilayer core as hypothesised in the past. ${ }^{899}$ Instead, for the polymer fragments investigated here, the composition does not correlate with the free energy of membrane disruption.

Because protonated polymers consistently interact better with lipid than ionised polymers in the free energy curves, it is intuitive to postulate polymers with intermediate level of ionisation would experience free energy barrier between the fully deprotonated and fully neutral form. Combining this with experimental results showing protonation is in competition with polymer precipitation, which destroys its membranelytic ability, ${ }^{9}$ it is possible that the higher membranelytic activity of grafted polymer is due to its capability to reach higher protonation 
state before precipitation occurs.

\begin{tabular}{|c|c|c|c|c|c|c|c|c|}
\hline $\begin{array}{c}\text { Free Energy } \\
(\mathrm{kJ} / \mathrm{mol})\end{array}$ & AAAAA & AAAAA & AABAA & AABAA & AAAAB & AAAAB & BBBBB & BBBBB \\
\hline \multirow{2}{*}{ Min } & -22.3 & -18.6 & -21.6 & -14.7 & -23.8 & -17.8 & -22.7 & -16.5 \\
& \pm 1.2 & \pm 1.4 & \pm 1.0 & \pm 2.2 & \pm 1.0 & \pm 0.6 & \pm 0.9 & \pm 0.9 \\
\hline \multirow{2}{*}{ Max } & 168 & 258 & 193 & 226 & 178 & 258 & 165 & 339 \\
& \pm 3.7 & \pm 4.8 & \pm 3.9 & \pm 6.1 & \pm 5.6 & \pm 2.7 & \pm 2.3 & \pm 2.6 \\
\hline \multirow{2}{*}{ Difference } & 190 & 277 & 214 & 241 & 202 & 276 & 187 & 355 \\
& \pm 3.9 & \pm 5.0 & \pm 4.0 & \pm 6.6 & \pm 5.7 & \pm 2.8 & \pm 2.5 & \pm 2.7 \\
\hline
\end{tabular}

Table 2: Free energy summaries for polymers of different compositions, either in the fully protonated or deprotonated states, showing the free energy minima reached at the water-membrane interface, the maxima towards the bilayer center and the difference between the maxima and minima.

In the above investigation, we have modelled the mechanism of membrane disruption as pore formation, which is amongst a number of possible mechanisms for membrane disruption. ${ }^{11146 / 47}$ One simulation study done by Yesylevsky et al. with natural peptide indicated that micropinocytosis might be an efficient pathway as compared with pore formation. $\stackrel{48}{ }$ However, accurate modelling micropinocytosis requires much bigger lipid systems and the study by Yesylevsky et al., only saw a possible onset of this process.

\subsection{Radial Distribution Functions (RDF)}

One aim of conformational analysis is to probe the polymer structure in aqueous solutions. We focused on polymer strands AAAAA and BBBBB, as they have the largest differences in composition, and investigated their conformational states in fully protonated and deprotonated states.

The RDF of the solvent environment distribution around polymers were computed. The $\mathrm{RDF}$ for a particular polymer was obtained from a single trajectory lasting $120 \mathrm{~ns}$, with the polymer solvated in a $6 \times 6 \times 6 \mathrm{~nm}^{3} \mathrm{SPC} / \mathrm{E}$ water cube, including the exact amount of buffer and counter ions to obtain an electroneutral system. The first $30 \mathrm{~ns}$ of the simulation was treated 


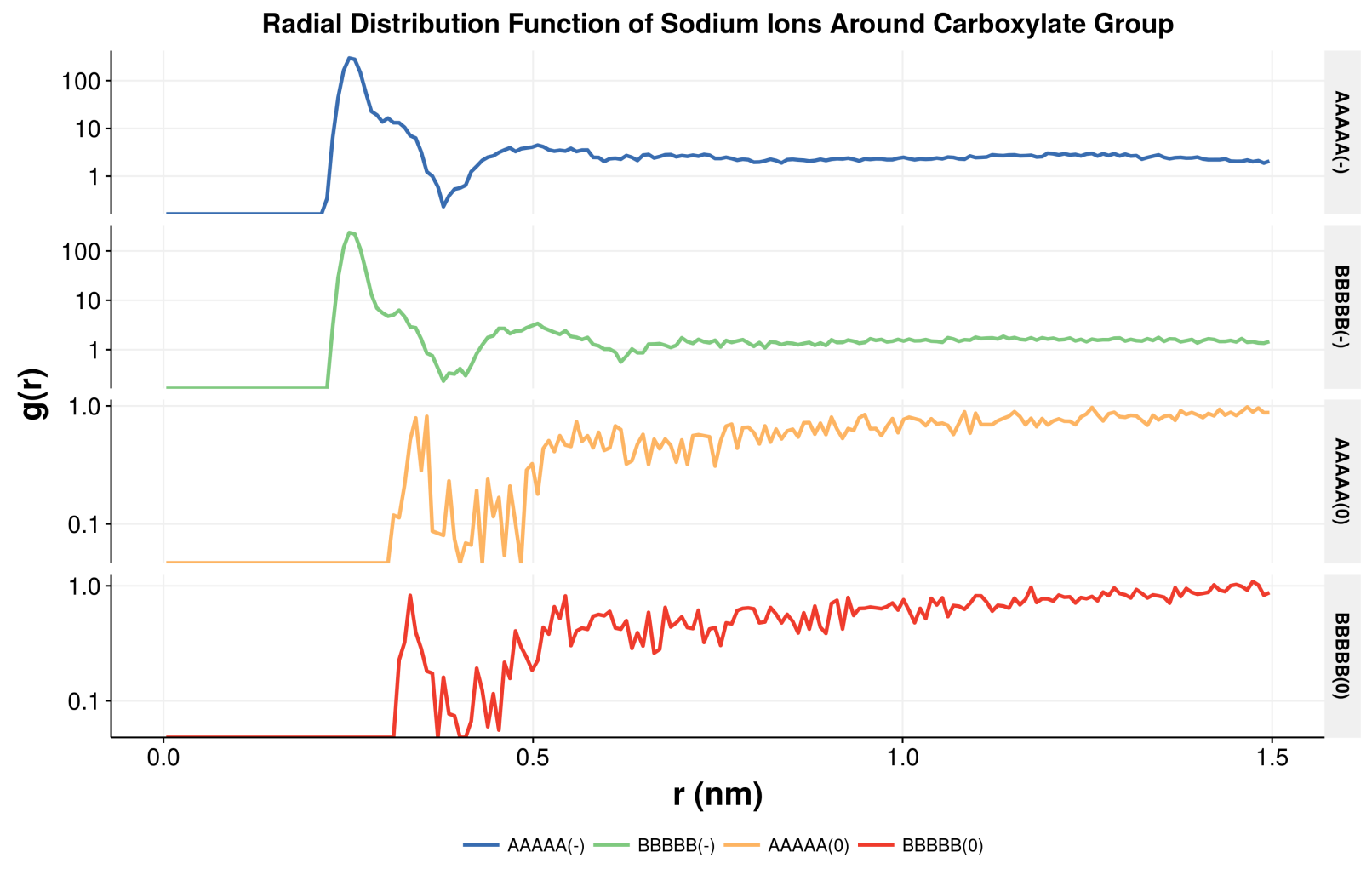

Figure 6: Radial distribution functions of $\mathrm{Na}^{+}$ion around (de-)protonated sites in the polymer. The plots show the radial distribution function $\mathrm{g}(\mathrm{r})$ between carboxylate group (highlighted in Fig. 2) carbon (either 'CO' or 'CN' carbon for type A and B monomer) and the $\mathrm{Na}^{+}$cations. The RDFs for each polymer were obtained from averaging the results from $1.5 \times 10^{3}$ frames spanning 30 ns. The $y$ axis is represented in log scale to facilitate the representation and comparison of the two top panels, where the maximum of the RDF reaches significant values of above 200 . 
as an equilibration run. The last $90 \mathrm{~ns}$ of simulation was divided into 3 parts and the RDFs of 1500 frames within each time-span were averaged to test the consistency and convergence of the radial distribution functions.

We first computed the RDF of water around the protonation site of the carboxylate groups shaded in figure 2, using oxygen for water and the 'CO' carbon for type A and 'CN' carbon for type B monomer . The RDFs feature a weak structure (see Figure 13 in the Supplementary Information), indicating absence of strong solvation around specific binding sites. Water features a slightly stronger structure when it is exposed to the BBBBB polymers, while changes in the protonation state of the polymer do not modify significantly this structure. This is similarly observed for the RDFs between the carboxylate groups and chloride ions (see Figure ?? in the supplementary information).

We then examined the RDF of sodium ions around the carboxylate groups (see Fig. 6). The profiles for the neutral polymers markedly fluctuate, but the RDF do not show prominent primary peaks close to the carboxylate groups, which would be indicative of strong binding. In addition, these RDFs are fairly insensitive to the grafting type (A or B). In comparison, the RDFs of the charged polymers show strong peaks at $\sim 0.3 \mathrm{~nm}$, which are indicative of strong Nacarboxylate binding. Inversion of the RDF via $F(r)=-k T \ln g(r)$, allows the estimation of the free energy, $F$, of ion adsorption, from $F\left(r_{\max }\right)-F(r \rightarrow \infty)$, where $r_{\max }$ is the distance at which we observe the first maximum in the RDF. The peak values of the RDFs for both charged polymers are above 250, with the peak for AAAAA(-) being slightly higher, which indicates a higher binding affinity for the ions compared to $\operatorname{BBBBB}(-)$. This is likely due to less steric hindrance from the side chain. This translates in a free energy of $\sim-5.5 \mathrm{k}$ for both polymers. Such binding free energies do not greatly affect how the charged polymers interact with the lipid head group as can be seen from the aforementioned PMF minima, and the ions feature a significant number of unbind and bind events, during our $>100 \mathrm{~ns}$ simulation trajectories.

Fig. 7 illustrates snapshots in the MD trajectory for the different polymers. In the neutral polymer cases, no discernible bias of either type of ions was observed near the polymer, which 
supports the conclusions from the RDF profiles. For the charged ones, both trajectories often show multiple sodium ions attracting to the carboxylate groups, at most one ion per carboxylate group. In Fig.7, both AAAAA(-) and BBBBB(-) are shown to have all five of its carboxylate groups occupied. However, no ions are ever encapsulated, which is reflected in the small free energy of adsorption estimated above. The short length of the polymer fragments can contribute to this, since this will limit them from adopting conformations where multiple carboxylate groups can point inwards to wrap sodium ions. Experiments with full length polymers have shown that

the polymers can trap pyrene, which is larger than the ions. ${ }^{8}$ The snapshots in Figure 7 also show that the $\mathrm{Cl}^{-}$anions never get too close to the polymers, a fact that is reflected in the $\mathrm{Cl}^{-}$carboxylate groups RDFs, which feature very little structure (see Figure 14 in the Supplementary Information). The snapshots represented in Figure 7, also show the presence of ion pairing near the polymer structure, irrespective of the protonation state and chemistry of the polymer (c.f. Figures 7-a and 7-d for AAAAA(0) and BBBBB(-) respectively.).

\subsection{Conformationally Stable States of Polymers}

In past computational studies, $\stackrel{[15] 19}{{ }^{2}}$ well-equilibrated time trajectories were used to extract shape descriptors such as radius of gyration and polymer end-to-end distances, without differentiating specific conformational states. We have employed here the MSM approach to identify the most long-lived conformation of each polymer in both water and at the water-lipid interface. For each of the four polymers, 8 parallel MD trajectories were run for $100 \mathrm{~ns}$, using the last frame of the aforementioned 120 ns simulation as the common starting configuration. The total 800 ns trajectory was then used for the Markov state modelling.

\section{Polymers in water}

From GROMACS generated trajectories, we extracted only the polymer from each frame (one frame per every $20 \mathrm{ps}$ ) and centered it. The first structure at time zero was chosen as the reference structure. We re-orientated every other structure in the trajectory against the reference 


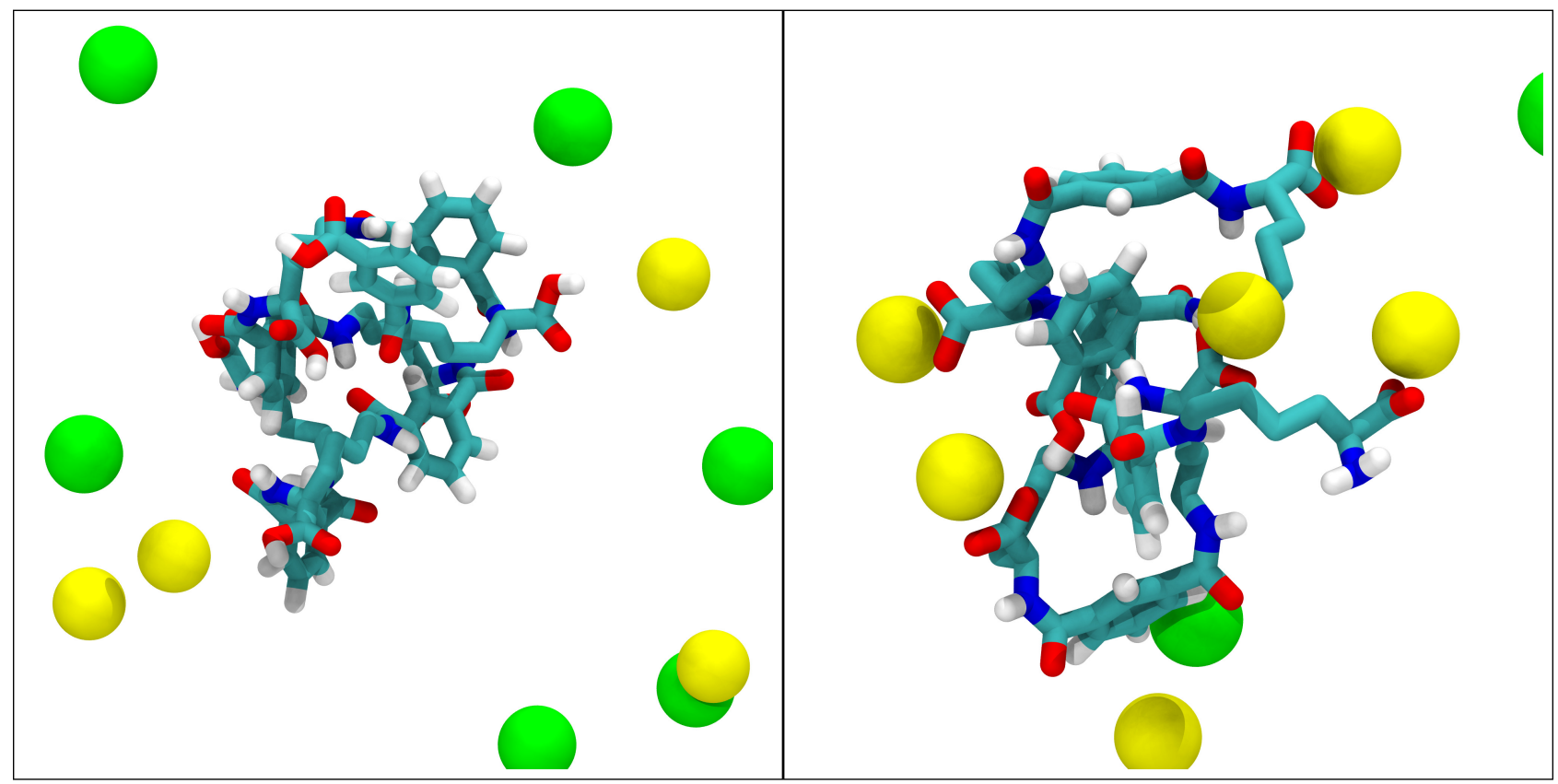

(a) AAAAA(0). No stable pairing with $\mathrm{Na}^{+}$ion observed

(b) AAAAA(-). Attracting multiple $\mathrm{Na}+$ ions by carboxylate group.

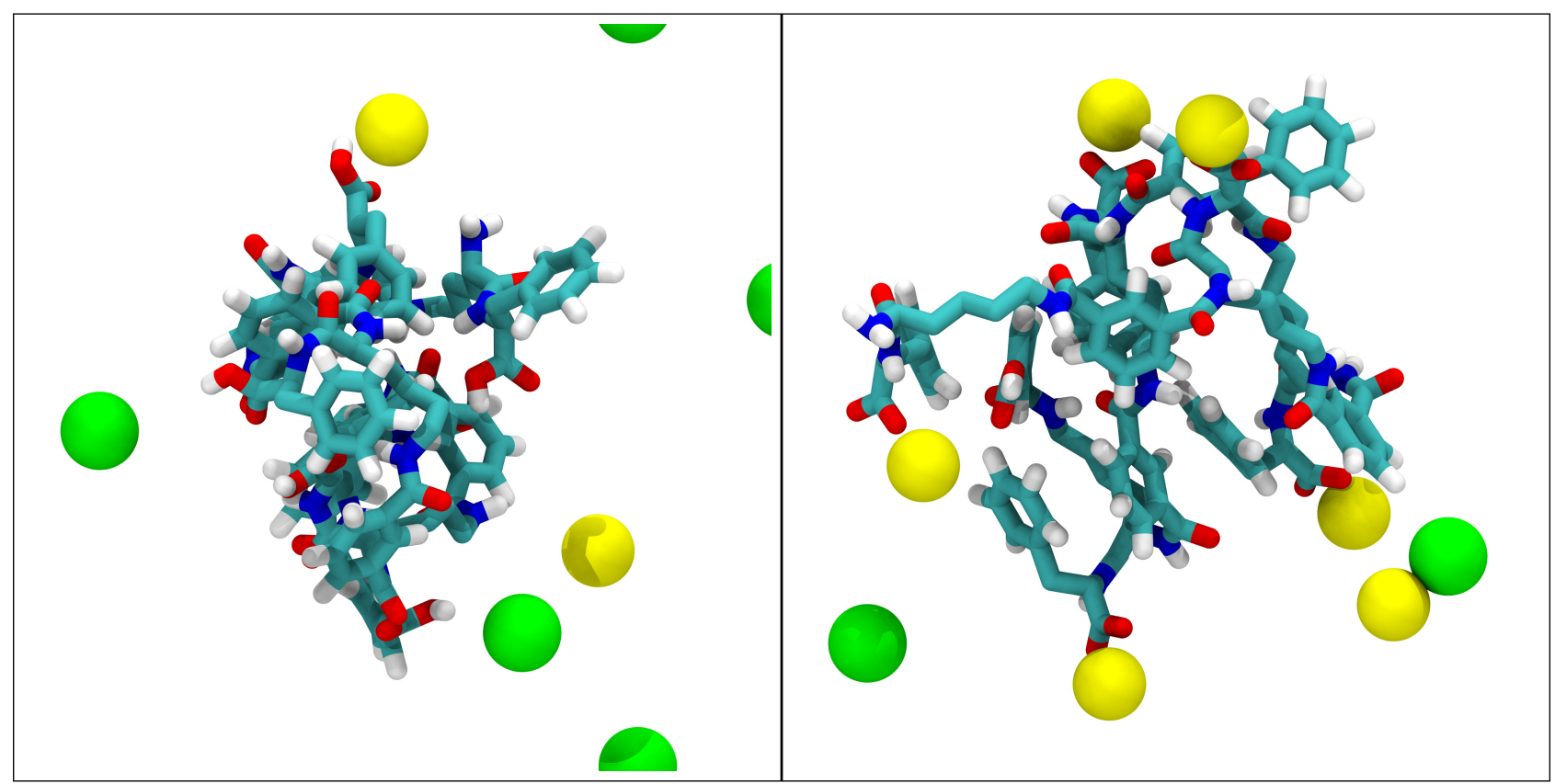

(c) $\mathrm{BBBBB}(0)$. No stable pairing with $\mathrm{Na}^{+}$ion observed (d) $\mathrm{BBBBB}(-)$. Attracting multiple $\mathrm{Na}+$ ions by carboxylate group.

Figure 7: Simulation frames showing the $\mathrm{Na}^{+}$ion distribution around the polymer. The polymers are shown in licorice format and the ions as spheres with $\mathrm{Na}^{+}$in yellow and $\mathrm{Cl}^{-}$in green. 
by minimising the root mean square distance between the coordinates of all atom pairs in a given structure and the reference. In this way, the difference in structures due to the rotation of the polymer fragments can be removed, and focus can be drawn on pure conformational differences. We built the MSM of the $\operatorname{BBBBB}(-)$ in water with a set of parameters that gave optimal convergence and the same tuning parameters were used for all other polymers. First, we used the $x y z$ coordinates of every second backbone atom in each monomer, namely CE1, CF, N1, CD, CB, N, as input descriptors (see Figure 2 for the meaning of these labels). These atoms are shared by all four types of monomers which allows ease of comparison and they are suitable for describing the backbone folding states of each polymer. We did not chose every atom in the backbone because the positions of adjacent atoms are heavily correlated. For each polymer, the procedure above resulted in 90 descriptors (i.e. the $x y z$ coordinates of 30 atoms). We used TICA to project these coordinates into lower dimensional spaces, by selecting top ranked columns from the diagonalised TICA eigenvector matrix that can describe $90 \%$ of the trajectory dynamics. The new number of descriptors was around 30 for each polymer.

Next, the coordinate space was discretised using the k-means algorithm. ${ }^{49}$ Through numerous trials for optimal convergence, 50 cluster centers were chosen. Implied time-scale analysis was performed using a list of lag times ranging from 10 up to 300 frames (frames were extracted every 20 ps). At small lag time, the decay time-scale (or correlation time) does not stay constant with increasing lag time and only flattens starting from a lag time of above 100 steps (see Figure 15 in the Supplementary Information). This indicates that the time correlation of the polymer starts to die out beyond 100 frames, or 2 ns separations. Following this analysis we selected a lag time of 200 frames to build the transition matrix.

Using PCCA, we sampled 100 structures belonging to the most stable conformational state for each polymer. Figure 8 lists the longest decay time for each polymer with a representative structure for that state. Representative structures were constructed by averaging the 100 structures to create an isosurface and overlaying on top one typical structure selected out of those 100. The colouring of the isosurface goes as red, orange, yellow, green, cyan, blue (rainbow 
colour except violet) with red indicating the N-terminus of the first monomer unit and blue the C-terminus of the last monomer.

Tracing the colours, one can see the most stable states of the polymers are all coiled. The polymers adopt a globular structure, while there is no significant difference on the level of folding upon protonation state change for both polymers. The calculated end to end distance distributions of the polymers peaks around $1.3 \mathrm{~nm}$ for all polymers irrespective of their charge state (fully extended polymer is over $5 \mathrm{~nm}$ ). Another common measure of the polymer conformation is the radius of gyration that characterises the average size of each polymer. Formally, it is defined as : $R_{g}=\sqrt{\frac{\sum_{i} m_{i}\left|\mathbf{r}_{i}-\mathbf{r}_{\text {com }}\right|^{2}}{\sum_{i} m_{i}}}$ where the sums iterate through every atom (including those not being used as descriptors for the MSMs) in the polymer with $\mathbf{r}_{i}$ and $m_{i}$ being the corresponding atomic coordinates and mass. $\mathbf{r}_{c o m}$ is the coordinate of the center of mass of the molecule.

Fig. 9 compares the radius of gyration for the polymers in water. It is intuitive to see that type A polymers have smaller sizes than type B polymers due to the less bulky side chain. Due to this, the charged form of A is still smaller than the neutral type B. Upon protonation, the gyration distribution for both neutral polymers are left-shifted. This is in accordance with the experimental findings, which attributed the cause to disappearance of electrostatic repulsions between carboxylate groups. ${ }^{9}$ However, the same experiment showed the charged polymers can adopt extended chain conformations. Our MSM structures do not show this trend. We believe that this can be due to the shorter size of the polymers investigated here and the impact of the polymer length on the limited electrostatic repulsion which may drive the formation of the more extended conformations. We find the size reduction is more pronounced for type A, the un-grafted polymer. Using the electrostatics argument, the less elaborate side chains of A offer less shielding of the charges. Combining end to end distance measure with the radius of gyration, we can infer the ends of polymer tend to point outwards rather than being buried inside the polymer core.

As shown in the figure 8, an important finding from the MSM is the gross difference in decay time across different polymer compositions. For type A, both charged states have similar decay 


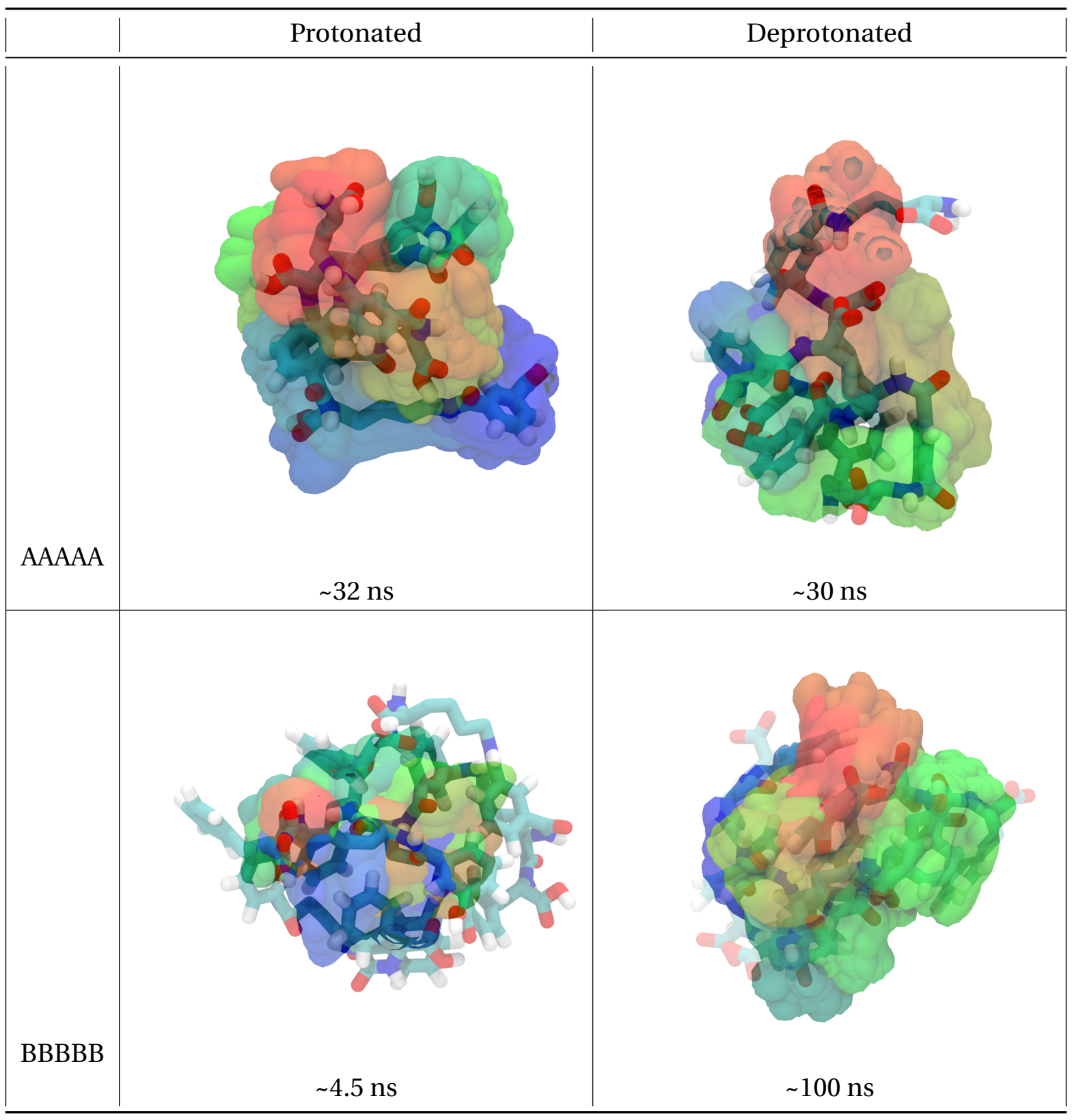

Figure 8: Longest lived conformations for each polymer in solution. The most stable conformer of each polymer of a charge state is shown. The isosurface is constructed from averaging 100 structures sampled using PCCA (from a total of $800 \mathrm{~ns}$ simulations). The colouring goes as red, orange, yellow, green, cyan, blue (rainbow colour order) starting from the N-terminus of the first monomer to the C-terminus of fifth unit. One representative structure is overlaid on top of the isosurface. The number underneath each figure is the decay time of the conformation estimated from the transition matrix. 
time of intermediate length. This indicates the change in $\mathrm{pH}$ might not affect the rate of rearrangement of the polymer. In comparison, for type $B$, the addition of phenylalanine side chains drastically stabilise the conformation of the charged form by 3 orders of magnitude. While in the protonated form, BBBBB is extremely prone to re-folding. Based on the results, we hypothesise the following. The ion affinities for both $\operatorname{AAAAA}(-)$ and BBBBB(-) are similar as shown in the RDFs (see Figure 6). But the large hydrophobic groups severely hinder BBBBB(-) to transition between low energy conformers. In the protonated case there is no ion affinity drive for conformers to stay put, but the hydrophobic chains increase the exposed area of the polymer to the solvent, which might favour quicker polymer rearrangements.

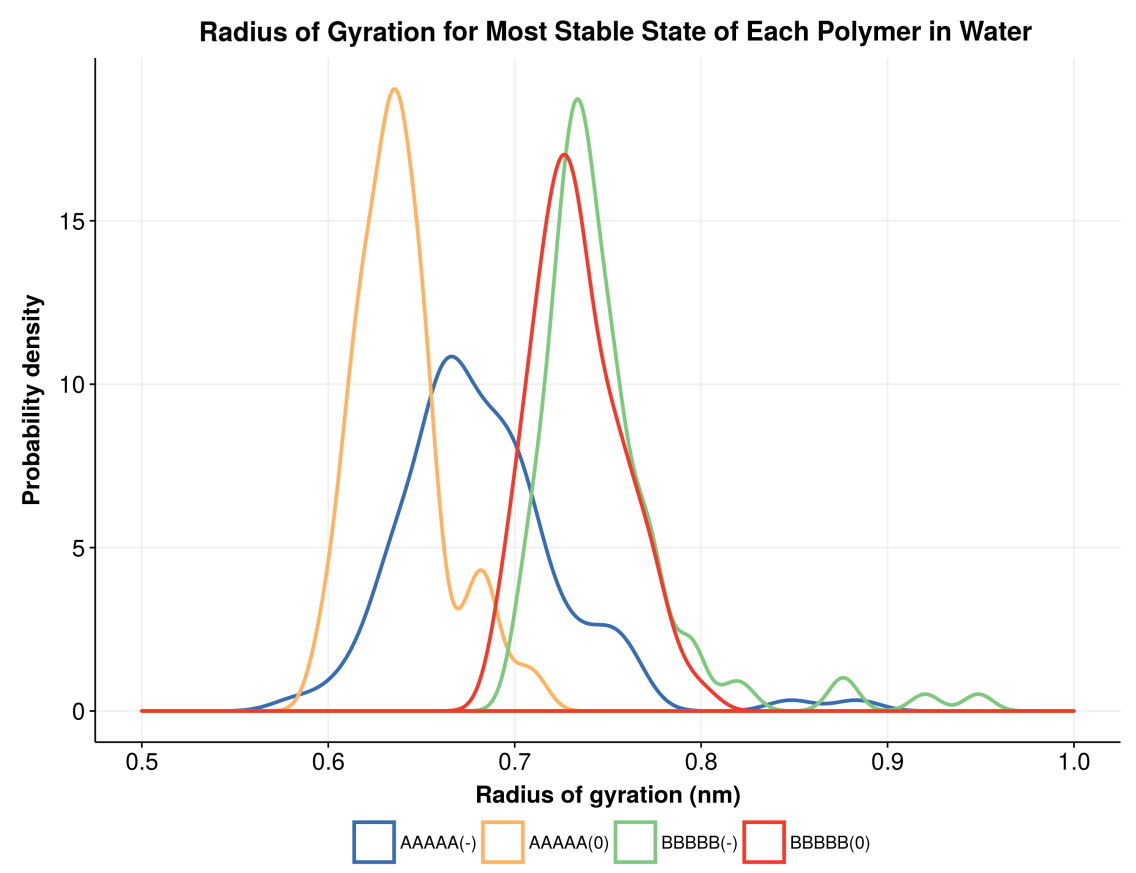

Figure 9: Radius of gyration distributions for stable conformations of polymers in water. The normalised distribution for each polymer is obtained from tallying 100 conformers from a total of 800 ns simulations.

\section{Polymers at the water-bilayer interface}

We then simulated the polymers residing at the water-lipid interface. As seen in the free energy, polymers like to be at the interface, hence additional simulations were performed with the poly- 
mers placed at the interface. This was done for each polymer by picking a starting frame from a simulation window obtained from previous umbrella sampling and running 30 ns simulation without restraining it. On average the polymer center of masses were $2 \mathrm{~nm}$ from the bilayer center of masses for the starting frames. We performed the same MSM analysis explained before for these new trajectories. This time, during TICA dimension reduction, only around ten descriptors were needed to describe $90 \%$ of the observed dynamics, suggesting that the presence of lipid head group greatly restricts the possible folds of the polymer backbones.

Fig. 10 depicts the radii of gyration. The size distributions now span a large range and all species experience a right-shift in peak compared to bulk water. This suggests the polymers adopt more extended conformations due to the presence of the lipid head groups. We find that $\mathrm{BBBBB}(-)$ has the largest radius of gyration (see Figure 10). This is consistent with the free energy minima observed before as the polymer is able to interact with the DPPC head groups at larger distance of separation. In the interfacial environment, the reduction in size due to pro-

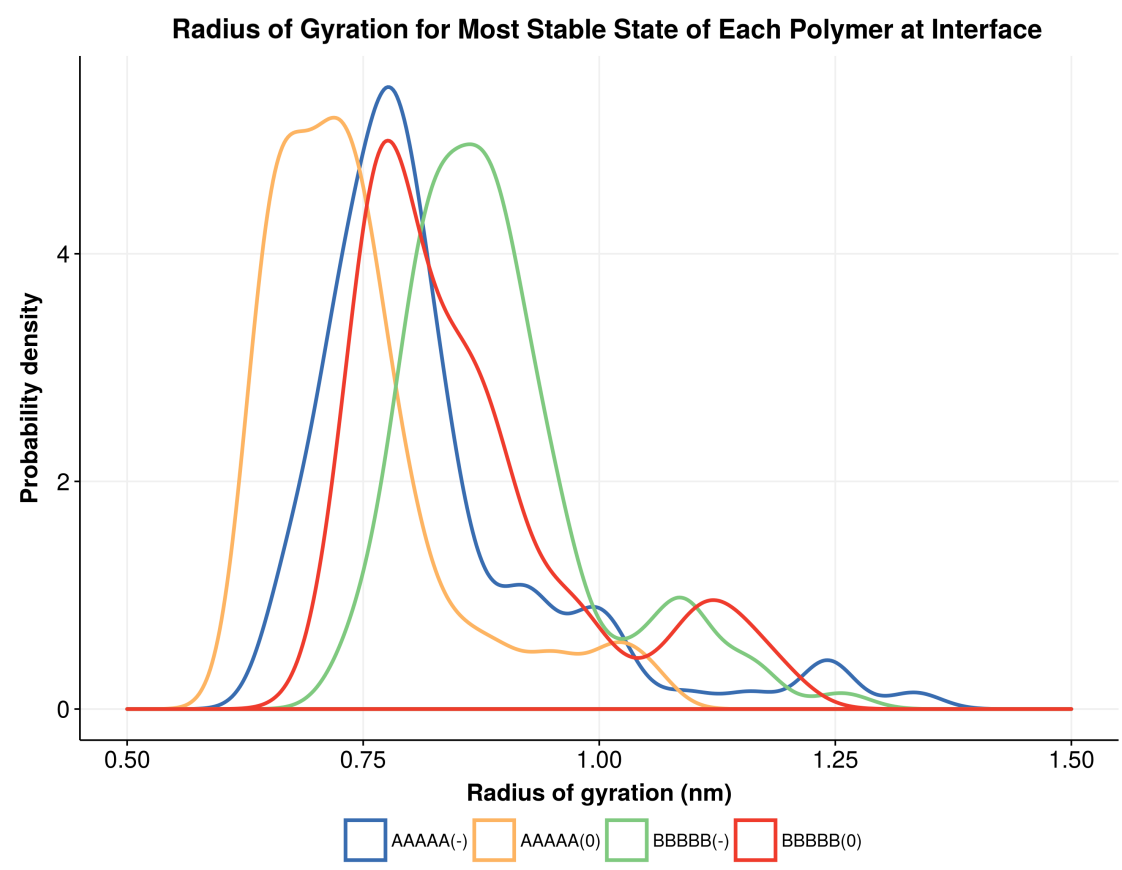

Figure 10: Radius of gyration distributions for stable conformation of polymers at water-lipid interface. The normalised distribution for each polymer is obtained from tallying 100 conformers obtained from a total of 800 ns simulations. 
tonation is more pronounced for both polymers. This is somewhat counter-intuitive. More extended structures should have the side chains further away from one another. In turn, the electrostatic repulsions felt would be reduced. Upon protonation, the weakening of charge repulsions should thus have less effect on the size of the polymer. One possible explanation is the positive amino groups of the lipid attract the neutral and charged polymer to different extend. This would manifest in the different level of preferential alignment of C-O vectors at the protonation sites with the lipid normal. We found little differences in the probability distribution of the $\mathrm{C}-\mathrm{O}$ vector orientation with respect to the bilayer plane.

Stable polymer conformations at the interface are shown in figure 11 . It is evident the backbones are more elongated in all cases. Consequently, the polymers have simpler folds, especially for the charged ones, where the isosurfaces show there is only one major bend approximately in the middle of both polymer chains. Comparison of the isosurface and the overlayed single structures indicate that the side chains of type B polymers tend to adhere to the backbone when the polymer is in bulk water, while at the interface, the side groups become more floppy and withhold from following the backbone trace.

The analysis of the isosurfaces for bulk water and the interface indicates a transition in the shape of the polymer, from coiled or globular to a less symmetric, rod-like form. An interesting question is whether this conformation change is driven by the interaction area with the membrane surface. We visually inspected the relative orientation of the polymers with the lipid surface. In the trajectories, the polymers tend to lie on the surface to increase the contact area. Fig. 12 shows a representative frame. This supports experimental findings on these polymers ${ }^{8}$ as well as the favourable free energy of interaction between polymer and lipid head group reported above.

The conformer decay times for all polymers are similar and short. This suggests the backbones can rapidly rearrange. Intuitively, making larger conformational changes is instrumental to tear open the bilayer. All but one polymer has much longer decay time in water than at interface - only $\operatorname{BBBBB}(0)$ has comparable decay time in water and at the interface. This 
hints at a possible kinetic drive for the observed better membranelysis ability of neutral type B. ${ }^{[8} \mathrm{BBBBB}(0)$ does not have conformational basin irrespective of the environment. Thus, it can most rapidly transition to the elongated form upon approaching the bilayer and initiate tearing.

\section{Conclusions}

In this study, we built the foundation for analysing a novel class of polymers with drug delivery potential, namely, poly(lysine iso-phthalamide) with and without phenylalanine grafted side chains. We constructed force field parameters for different monomer units and defined rules for concatenating monomers. With such implementation, polymers of various length and compositions can be simulated.

We then focused on fully protonated and deprotonated species and calculated their free energy changes during the membrane penetration process. We found the protonated forms incurred less energetic penalties than the deprotonated ones in order to reach the lipid core. This is in support of experimental findings that membranelysis by these polymers occur at lowered $\mathrm{pH}$ in the cell. When fully protonated, the free energy minima and maxima experienced by the grafted and un-grafted polymers were found to be very similar. This suggests the experimentally observed better membranelytic capability of grafted polymer was possibly mainly due to its ability to achieve higher level of protonation before precipitation.

MSM was used for the first time to study drug delivery molecules. It provided a molecular level understanding of the fold states of short polymer strands. All polymers became more elongated going from water to the membrane surface to gain interaction surface with the lipid head groups. From time-scale analysis using MSM, the protonated grafted polymer was the only species that had comparably short conformational transition time (on the order of a few nanoseconds) in water and at the interface. We hypothesise that its ability to most rapidly switch from its water phase conformation to that at interface is a possible kinetic factor for the observed better performance. 


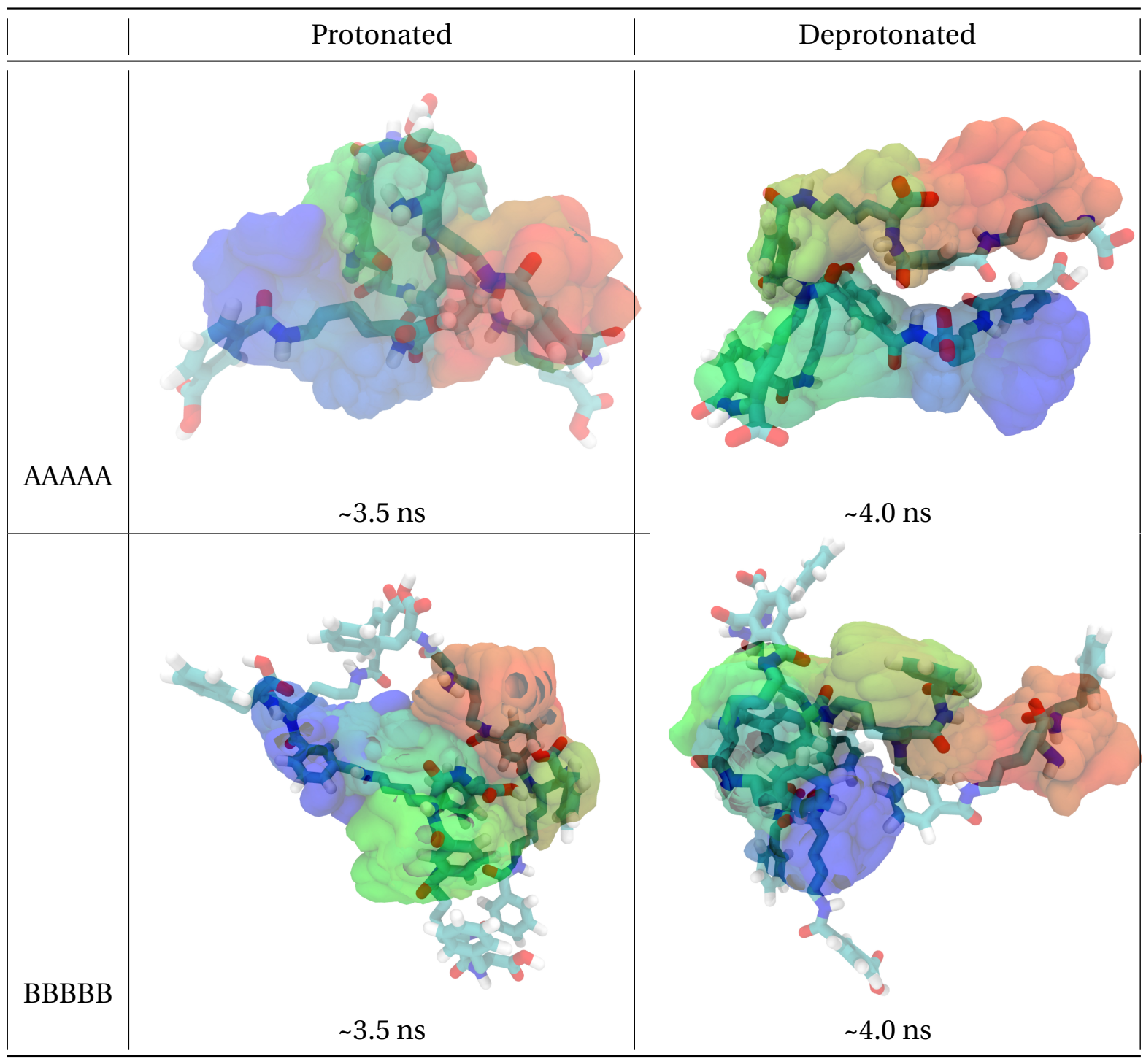

Figure 11: Longest lived conformations for each polymer at lipid-water interface. The most stable conformer of each polymer of a charge state is shown. The isosurface is constructed from averaging 100 structures (from a total of $800 \mathrm{~ns}$ simulations) sampled using PCCA. The colouring goes as red, orange, yellow, green, cyan, blue (rainbow colour order) starting from the $\mathrm{N}$-terminus of the first monomer to the C-terminus of fifth unit. One representative structure is overlaid on top of the isosurface. The number underneath each panel represents the decay time of the conformation estimated from the transition matrix. 


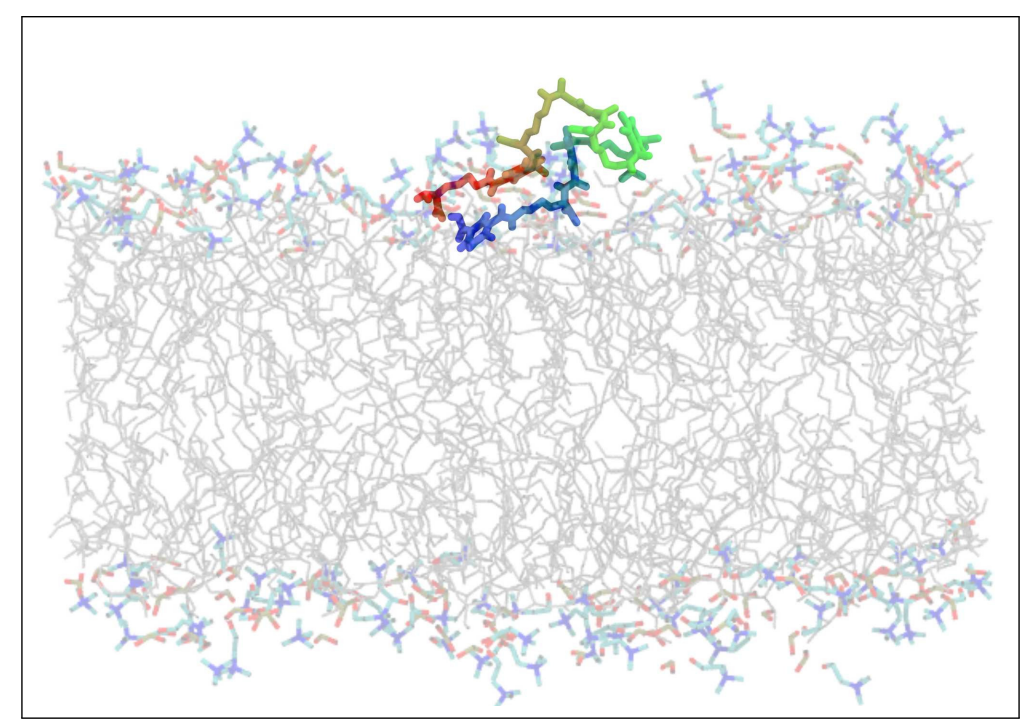

Figure 12: Illustration of relative orientation of polymer AAAAA(-) with the lipid surface. The lipid bilayer is represented semi-transparently for clarity. The polymer strand is coloured the same order as the isosurface representations in Figure 11.

Further works can be performed in a number of directions. The primary investigation should be on calculating PMFs for partially protonated species for type A and B polymers. This will confirm whether free energies paths are indeed between those observed for the fully protonated and deprotonated forms. If so, this will consolidate the level of protonation as the main cause of membranelytic ability. In addition, the same analysis can be run on polymers with different combinations of $\mathrm{A}$ and $\mathrm{B}$, or on polymers with other amino acid grafts after constructing their force fields.

With increased system sizes and longer simulation time, we could approximate the experimentally observed polymer cooperativity by simulating multiple strands and observe interpolymer interactions. Simulation of polymers with more repeat units can better mimic larger scale polymer arrangements.

\section{Acknowledgement}

The authors thank Dr. Rongjun Chen for insightful discussions. SW thanks Anna Sofia Tascini, Silvia Di Lecce and Dr. Miguel Gonzalez for their help with force field parametrisation, HPC 
set-ups and free energy calculations. We thanks EPSRC-UK (EP/J003859/1, EP/J017566/1) and EU 7th Framework Programme ITN-SNAL for financial support and the Imperial College High Performance Computing Service for providing computational resources.

The supplementary information includes description to the force field constructed for the monomers, radial distribution function of water and chloride ions around the carboxylate groups of the polymers and an example plot for implied time-scale analysis.

\section{References}

(1) DiMasi, J. A.; Grabowski, H. G.; Hansen, R. W. Innovation in the pharmaceutical industry: New estimates of R\&D costs. J. Health Econ. 2016, 47, 20-33.

(2) Huynh, L.; Neale, C.; Allen, C. Computational approaches to the rational design of nanoemulsions, polymeric micelles, and dendrimers for drug delivery. Nanomedicine: Nanotechnology, Biology, and Medicine 2012, 8, 20-36.

(3) Huang, Y.; Wang, D.; Zhu, X.; Yan, D.; Chen, R. Synthesis and therapeutic applications of biocompatible or biodegradable hyperbranched polymers. Polym. Chem. 2015, 6, 27942812.

(4) Liu, J.; Huang, W.; Pang, Y.; Zhu, X.; Zhou, Y.; Yan, D. Self-assembled micelles from an amphiphilic hyperbranched copolymer with polyphosphate arms for drug delivery. Langmuir 2010, 26, 10585-10592.

(5) Bunker, A. Poly(Ethylene Glycol) in Drug Delivery, Why Does it Work, and Can We do Better? All Atom Molecular Dynamics Simulation Provides Some Answers. Phys. Procedia 2012, 34, 24-33.

(6) Wang, S.; Liu, X.; Villar-Garcia, I. J.; Chen, R. Amino Acid Based Hydrogels with Dual Responsiveness for Oral Drug Delivery. Macromol. Biosci. 2016, 16, 1258-1264. 
(7) El-Sayed, M. E.; Hoffman, A. S.; Stayton, P. S. Smart polymeric carriers for enhanced intracellular delivery of therapeutic macromolecules. Expert Opin. Biol. Ther. 2015, 5, 23-32.

(8) Chen, R.; Eccleston, M. E.; Yue, Z.; Slater, N. K. H. Synthesis and pH-responsive properties of pseudo-peptides containing hydrophobic amino acid grafts. J. Mater. Chem. 2009, 19, $4217-4224$.

(9) Chen, R.; Khormaee, S.; Eccleston, M. E.; Slater, N. K. H. Effect of L-leucine graft content on aqueous solution behavior and membrane-lytic activity of a pH-responsive pseudopeptide. Biomacromolecules 2009, 10, 2601-2608.

(10) Eccleston, M. E.; Kuiper, M.; Gilchrist, F. M.; Slater, N. K. H. pH-responsive pseudopeptides for cell membrane disruption. J. Control. Release 2000, 69, 297-307.

(11) Yessine, M. A.; Leroux, J. C. Membrane-destabilizing polyanions: Interaction with lipid bilayers and endosomal escape of biomacromolecules. Adv. Drug Deliv. Rev. 2004, 56, 9991021.

(12) Chen, S.; Wang, S.; Kopytynski, M.; Bachelet, M.; Chen, R. Membrane-Anchoring, CombLike Pseudopeptides for Efficient, pH-Mediated Membrane Destabilization and Intracellular Delivery. ACS Appl. Mater. Interfaces 2017, 9, 8021-8029.

(13) Schulz, M.; Olubummo, A.; Binder, W. H. Beyond the lipid-bilayer: interaction of polymers and nanoparticles with membranes. Soft Matter 2012, 8, 4849-4864.

(14) Rossi, G.; Monticelli, L. Modeling the effect of nano-sized polymer particles on the properties of lipid membranes. J. Phys. Condens. Matter 2014, 26, 503101.

(15) Choudhury, C. K.; Kumar, A.; Roy, S. Characterization of conformation and interaction of gene delivery vector polyethylenimine with phospholipid bilayer at different protonation state. Biomacromolecules 2013, 14, 3759-3768. 
(16) Kepczynski, M.; Jamróz, D.; Wytrwal, M.; Bednar, J.; Rzad, E.; Nowakowska, M. Interactions of a hydrophobically modified polycation with zwitterionic lipid membranes. Langmuir 2012, 28, 676-688.

(17) Kim, Y.; Kwak, Y.; Chang, R. Free energy of PAMAM dendrimer adsorption onto model biological membranes. J. Phys. Chem. B 2014, 118, 6792-6802.

(18) Marrink, S. J.; Risselada, H. J.; Yefimov, S.; Tieleman, D. P.; De Vries, A. H. The MARTINI force field: Coarse grained model for biomolecular simulations. J. Phys. Chem. B 2007, $111,7812-7824$.

(19) Lee, H.; Larson, R. G. Molecular dynamics simulations of PAMAM dendrimer-induced pore formation in DPPC bilayers with a coarse-grained model. J. Phys. Chem. B 2006, 110, $18204-18211$.

(20) Nawaz, S.; Redhead, M.; Mantovani, G.; Alexander, C.; Bosquillon, C.; Carbone, P. Interactions of PEO-PPO-PEO block copolymers with lipid membranes: a computational and experimental study linking membrane lysis with polymer structure. Soft Matter 2012, 8, $6744-6754$.

(21) Samanta, S.; Hezaveh, S.; Roccatano, D. Theoretical study of binding and permeation of ether-based polymers through interfaces. J. Phys. Chem. B 2013, 117, 14723-14731.

(22) Oostenbrink, C.; Villa, A.; Mark, A. E.; Van Gunsteren, W. F. A biomolecular force field based on the free enthalpy of hydration and solvation: The GROMOS force-field parameter sets 53A5 and 53A6. J. Comput. Chem. 2004, 25, 1656-1676.

(23) De Nicola, A.; Hezaveh, S.; Zhao, Y.; Kawakatsu, T.; Roccatano, D.; Milano, G. Micellar drug nanocarriers and biomembranes: how do they interact? Phys. Chem. Chem. Phys. 2014, $16,5093-105$. 
(24) Tieleman, P. Biocomputing/University of Calgary.http://wcm . ucalgary.ca/tieleman/ downloads, (visited : 2017-04-23).

(25) Berendsen, H. J. C.; Grigera, J. R.; Straatsma, T. P. The Missing Term in Effective Pair Potentials. J. Phys. Chem. 1987, 91, 6269-6271.

(26) Dulbecco, R.; Vogt, M. Plaque formation and isoltion of pure lines with poliomyelitis viruses. J. Exp. Med. 1954, 99, 167-182.

(27) Bussi, G.; Donadio, D.; Parrinello, M. Canonical sampling through velocity rescaling. J. Chem. Phys. 2007, 126, 014101.

(28) Parrinello, M.; Rahman, A. Polymorphic transitions in single crystals: A new molecular dynamics method. J. Appl. Phys. 1981, 52, 7182-7190.

(29) Nosé, S.; Klein, M. Constant pressure molecular dynamics for molecular systems. Mol. Phys. 1983, 50, 1055-1076.

(30) Hess, B.; Bekker, H.; Berendsen, H. J. C.; Fraaije, J. G. E. M. LINCS: A linear constraint solver for molecular simulations. J. Comput. Chem. 1997, 18, 1463-1472.

(31) Darden, T.; York, D.; Pedersen, L. Particle mesh Ewald: An N log(N) method for Ewald sums in large systems. J. Chem. Phys. 1993, 98, 10089-10092.

(32) Gonzalez, M. A.; Barriga, H. M. G.; Richens, J. L.; Law, R. V.; O 'shea, P.; Bresme, F. How does Ytterbium chloride interact with DMPC bi- layers? a computational and experimental study. Phys. Chem. Chem. Phys. 2017, 19, 9199-9209.

(33) Van Der Spoel, D.; Lindahl, E.; Hess, B.; Groenhof, G.; Mark, A. E.; Berendsen, H. J. C. GROMACS: Fast, flexible, and free. 2005.

(34) Bekker, H.; Berendsen, H. J. C.; Dijkstra, E. J.; Achterop, S.; van Drunen, R.; van der Spoel, D.; Keegstra, H.; Reitsma, B.; Renardus, M. K. R. Gromacs: A Parallel Computer for Molecular Dynamics Simulations. Physics Computing'92 1993, 252-256. 
(35) Berendsen, H. J. C.; van der Spoel, D.; van Drunen, R. GROMACS: A message-passing parallel molecular dynamics implementation. Comput. Phys. Commun. 1995, 91, 43-56.

(36) Humphrey, W.; Dalke, A.; Schulten, K. VMD: Visual molecular dynamics. J. Mol. Graph. 1996, 14, 33-38.

(37) High Performance Computing (HPC) | Imperial College London. http://www . imperial. ac.uk/admin-services/ict/self-service/research-support/hpc/, (visited : 201705-05).

(38) Prinz, J. H.; Wu, H.; Sarich, M.; Keller, B.; Senne, M.; Held, M.; Chodera, J. D.; Schtte, C.; Noé, F. Markov models of molecular kinetics: Generation and validation. J. Chem. Phys. 2011, 134, 1-23.

(39) Prinz, J.-H.; Keller, B.; Noe, F. Probing molecular kinetics with Markov models: metastable states, transition pathways and spectroscopic observables. Phys. Chem. Chem. Phys. 2011, $13,16912-16927$.

(40) Schwantes, C. R.; Pande, V. S. Improvements in Markov State Model construction reveal many non-native interactions in the folding of NTL9. J. Chem. Theory Comput. 2013, 9, 2000-2009.

(41) Pérez-Hernández, G.; Paul, F; Giorgino, T.; De Fabritiis, G.; Noé, F. Identification of slow molecular order parameters for Markov model construction. J. Chem. Phys. 2013, 139, 015102.

(42) Forgy, E. Cluster Analysis of Multivariate Data: Efficiency versus Interpretability of Classification. Biometrics 1965, 21, 768-769.

(43) Swope, W. C.; Pitera, J. W.; Suits, F. Describing Protein Folding Kinetics by Molecular Dynamics Simulations. 1. Theory. J. Phys. Chem. B 2004, 108, 6571-6581. 
(44) Deuflhard, P.; Weber, M. Robust Perron cluster analysis in conformation dynamics. Linear Algebra Appl. 2005, 398, 161-184.

(45) Scherer, M. K.; Trendelkamp-Schroer, B.; Paul, F.; Hoffmann, M.; Plattner, N.; Wehmeyer, C.; Prinz, J. H.; Noe, F. PyEMMA 2: A Software Package for Estimation, Validation, and Analysis of Markov Models. J. Chem. Theory Comput. 2015, 11, 5525-5542.

(46) Linhardt, J. G.; Tirrell, D. A. pH-induced fusion and lysis of phosphatidylcholine vesicles by the hydrophobic polyelectrolyte poly(2-ethylacrylic acid). Langmuir 2000, 16, 122-127.

(47) Matsuzaki, K.; Yoneyama, S.; Miyajima, K. Pore formation and translocation of melittin. Biophys. J. 1997, 73, 831-838.

(48) Yesylevskyy, S.; Marrink, S. J.; Mark, A. E. Alternative mechanisms for the interaction of the cell-penetrating peptides penetratin and the TAT peptide with lipid bilayers. Biophys. J. 2009, 97, 40-49.

(49) Hadley, K. R.; McCabe, C. A Simulation Study of the Self-Assembly of Coarse-Grained Skin Lipids. Soft Matter 2012, 8, 4802-4814. 